\title{
Fat bundles and formality
}

\author{
by Wojciech Andrzejewski and Aleksy Tralle (Szczecin)
}

\begin{abstract}
We prove the formality property of total spaces of fat bundles over compact homogeneous spaces. Some rational homotopy obstructions to fatness are obtained.

1. Introduction. It is well known that any compact Kähler manifold is formal [3]. A simple argument with harmonic forms shows that any Riemannian symmetric space is formal [8]. In the present paper we prove the "[3]type" theorem for fat bundles. There are examples of compact symplectic manifolds which are non-formal and hence carry no Kählerian structure [9]. Nevertheless, there are no examples of simply connected symplectic compact manifolds which are non-formal. The authors of [9] conjectured that any compact simply connected symplectic manifold is formal. They proved some theorems in this direction, and noticed the "formalizing tendency" of symplectic structures. An immediate difficulty encountered with such questions is the lack of examples (the familiar examples, such as $C P^{n}$ etc., are too simple to exhibit anything new).

On the other hand, there is a family of non-trivial compact symplectic manifolds, namely, the total spaces of associated fat bundles [14]. We contribute to the "formalizing tendency" by proving that fat bundles over compact homogeneous spaces are formal. Of course this result is of independent interest, because it describes the rational homotopy type and yields a new obstruction to the existence of fat bundles. In addition, the paper contains some algebraic results about Koszul complexes and twisted tensor products of Koszul complexes, associated to symmetric $P$-algebras. These results are useful in the rational homotopy theory of homogeneous spaces (see e.g. [1]). Various examples of fatness were given by A. Weinstein [14] and L. Bérard-Bergery [2]. Nevertheless, it seems that there were no explicit examples of $\mathcal{O}$-fatness, which is necessary for producing new symplectic structures $(\mathcal{O}$ denotes a co-adjoint orbit). We analyze $\mathcal{O}$-fatness, proving its
\end{abstract}

1991 Mathematics Subject Classification: 53C15, 55P62.

Key words and phrases: fat bundle, formality, symplectic structure. 
existence in some universal $S O(n)$-bundles. The main results of the paper are formulated below.

TheOREM 1.1. Let $P(M, G)$ be a principal bundle of a compact semisimple Lie group $G$ and $\mathcal{O}$ be any co-adjoint orbit of $G$ in the dual space $g^{*}$ to its Lie algebra $g$. Let $E=P \times_{G} \mathcal{O}$ be the total space of the associated bundle. If $P(M, G)$ is $\mathcal{O}$-fat, and $H / K=M$ is a compact homogeneous space of a compact semisimple Lie group $K$, then

(i) $E$ is a compact symplectic manifold which is formal;

(ii) $M$ is formal and $\operatorname{rank} H=\operatorname{rank} K$.

In the sequel we use the notion of a pure graded differential algebra introduced in [9]. This notion turned out to be important in symplectic geometry (see Section 4 for the definition of pureness).

Theorem 2.4. Let $\left(S, d_{S}\right)=\left(\bigvee Q \otimes \wedge P, d_{S}\right)$ and $\left(T, d_{T}\right)=\left(\bigvee Q^{\prime} \otimes\right.$ $\left.\bigwedge P^{\prime}, d_{T}\right)$ be two Koszul complexes, associated with the appropriate $P$-algebras. Let $\tau$ be any "transgression" map $\tau: P^{\prime} \rightarrow Z(S)$ such that $d_{\tau}$ : $S \otimes T \rightarrow S \otimes T$ defined by the formulae

$$
\left.d_{\tau}\right|_{S}=d_{S},\left.\quad d_{\tau}\right|_{Q^{\prime}}=0,\left.\quad d_{\tau}\right|_{P^{\prime}}=\left.d_{\tau}\right|_{P^{\prime}}+\left.\tau\right|_{P^{\prime}}
$$

is a derivation. Suppose that

$$
\operatorname{dim} Q^{\prime}=\operatorname{dim} P^{\prime} .
$$

If the twisted tensor product $\left(S \otimes_{\tau} T, d_{\tau}\right)$ has finite-dimensional cohomology algebra $H^{*}\left(S \otimes_{\tau} T, d_{\tau}\right)$, then it is pure.

THEOREM 5.1. Let $S(2 m+2 n, 2 m, 2 n) \stackrel{S O(2 n)}{\longrightarrow} \operatorname{Gr}(2 m+2 n, 2 m, 2 n)$ denote the universal $S O(2 n)$-bundle of a Stiefel manifold $S O(2 m+2 n) /$ $S O(2 m)$ over a Grassmann manifold $S O(2 m+2 n) / S O(2 m) \times S O(2 n)$ $(n>1)$. There exists a co-adjoint orbit $\mathcal{O}(\xi) \subset$ so $(2 n)^{*}$ such that the canonical connection of the Riemannian symmetric space $\operatorname{Gr}(2 m+2 n, 2 m, 2 n)$ is $\mathcal{O}(\xi)$-fat

Observe that Theorem 1.1 describes the rational homotopy type of fat bundles over compact homogeneous spaces and gives new obstructions to the existence of $\mathcal{O}$-fat connections (compare [14]). As far as Theorem 2.6 is concerned, it can be viewed as a sharpening of the Thomas theorem concerning the pureness of Serre fibrations [11]. The Thomas definition of pureness is weaker than the definition provided in this paper. Taking a Koszul complex as a base of the appropriate algebraic fibration, we impose an additional assumption but strengthen the pureness result.

The paper is organized as follows. Section 2 deals with the algebraic part of the paper, devoted to minimal models, $P$-algebras and homogeneous spaces. Section 3 describes the construction of fat bundles. Section 4 is 
devoted to the proof of the formality of fat bundles. Section 5 contains the explicit description of $\mathcal{O}$-fat connections. All preliminary material is given in each section separately.

2. Koszul complexes and minimal models of homogeneous spaces. Since there are many papers and books on rational homotopy theory (e.g. $[3,6,8,9])$, we assume the reader to be familiar with it. The proofs in this paper are based on the techniques of Koszul complexes and Cartan algebras. A more detailed exposition of this topic can be found in [14]. We consider the category $\mathbb{R}-\mathrm{DGA}_{(\mathrm{c})}$ of graded commutative differential algebras over the reals and suppose all the differentials to be of degree +1 . We say that two graded differential algebras $\left(\mathcal{A}, d_{\mathcal{A}}\right),\left(\mathcal{B}, d_{\mathcal{B}}\right) \in \mathbb{R}-\mathrm{DGA}_{(\mathrm{c})}$ are c-equivalent if there is a chain of algebras $\left(\mathcal{A}_{i}, d_{\mathcal{A}_{i}}\right) \in \mathbb{R}-\mathrm{DGA}_{(\mathrm{c})}, i=1, \ldots, k$, starting from $\left(\mathcal{A}, d_{\mathcal{A}}\right)=\left(\mathcal{A}_{1}, d_{\mathcal{A}_{1}}\right)$ and ending with $\left(\mathcal{A}_{k}, d_{\mathcal{A}_{k}}\right)=\left(\mathcal{B}, d_{\mathcal{B}}\right)$ such that each pair $\left(\left(\mathcal{A}_{i}, d_{\mathcal{A}_{i}}\right),\left(\mathcal{A}_{i+1}, d_{\mathcal{A}_{i+1}}\right)\right)$ is related either by a morphism

$$
\left(\mathcal{A}_{i}, d_{\mathcal{A}_{i}}\right) \rightarrow\left(\mathcal{A}_{i+1}, d_{\mathcal{A}_{i+1}}\right)
$$

or by a morphism

$$
\left(\mathcal{A}_{i+1}, d_{\mathcal{A}_{i+1}}\right) \rightarrow\left(\mathcal{A}_{i}, d_{\mathcal{A}_{i}}\right)
$$

inducing an isomorphism in cohomology. A morphism inducing an isomorphism on the cohomology level is called a quasiisomorphism. Any graded differential algebra $\left(\mathcal{A}, d_{\mathcal{A}}\right)$ that we consider satisfies $H^{0}\left(\mathcal{A}, d_{\mathcal{A}}\right)=\mathbb{R}$ and $H^{n}\left(\mathcal{A}, d_{\mathcal{A}}\right)$ is a finite-dimensional vector space for each $n$. We denote the ideal of positive degree elements in $\mathcal{A}$ by $\mathcal{A}^{+}$. If $V$ is a vector space, then $\bigwedge V$ denotes the free graded commutative algebra generated by $V$. If $\left\{v_{1}, v_{2}, \ldots\right\}$ is a basis for $V$, then we write $V=\left\langle v_{1}, v_{2}, \ldots\right\rangle$ and $\bigwedge V=\bigwedge\left(v_{1}, v_{2}, \ldots\right)$. A graded differential algebra is minimal if (1) $\mathcal{A} \simeq \bigwedge V$ for some $V$ and (2) there is a basis $V=\left\langle v_{1}, v_{2}, \ldots\right\rangle$ such that, for each $j, d v_{j} \in\left(\bigwedge\left(v_{1}, \ldots\right.\right.$ $\left.\left.\ldots, v_{j-1}\right)\right)^{+}\left(\bigwedge\left(v_{1}, \ldots, v_{j-1}\right)\right)^{+}$. We say that $(\bigwedge V, d) \in \mathbb{R}-\mathrm{DGA}_{(\mathrm{c})}$ is a minimal model of $\left(\mathcal{A}, d_{\mathcal{A}}\right)$ if there is a quasiisomorphism

$$
\varrho:(\bigwedge V, d) \rightarrow\left(\mathcal{A}, d_{\mathcal{A}}\right)
$$

We use the following

Proposition 1 [8]. Any two c-equivalent graded differential algebras have isomorphic minimal models.

In this paper we consider only smooth manifolds and their "real minimal models". That is, for any smooth manifold $M$ we call the graded differential algebra $\mathcal{M}_{\mathcal{E}}$ (which is the minimal model of the de Rham algebra $\mathcal{E}$ of $M$ ) the minimal model of $M$. We use the notation

$$
\mathcal{M}_{M}=\mathcal{M}_{\mathcal{E}}
$$


By definition, we say that a minimal algebra $(\bigwedge V, d)$ is formal if it is $c$-equivalent to its cohomology algebra $H^{*}(\bigwedge V, d)$. A manifold $M$ is called formal if $\mathcal{M}_{M}$ is formal.

Remark. Of course, it is enough for our purposes to use the above notions, but [3, 6-8] contain a more subtle topological approach.

In what follows we consider $P$-algebras and their Koszul complexes. In the sequel, $\bigwedge P$ denotes the exterior algebra over a finite-dimensional graded vector space $P=\bigoplus_{k} P^{k}$, graded by odd degrees. If $Q$ denotes an evenly graded vector space, then we use the notation $\bigvee Q$ for the symmetric algebra over $Q$.

Definition. A P-algebra is a pair $(S, \sigma)$, where:

(i) $S$ is a positively graded associative algebra with identity,

(ii) $P=\bigoplus_{k} P^{k}$ is a finite-dimensional positively graded vector space which satisfies the condition $P^{k}=0$ if $k$ is even,

(iii) $\sigma: P \rightarrow S$ is a linear mapping, homogeneous of degree 1 , which satisfies

$$
\sigma(x) z=z \sigma(x), \quad x \in P, z \in S .
$$

The following graded differential algebra is associated with each $P$-algebra $S$. In the tensor product $S \otimes \wedge P$ define a linear operator $\nabla_{S}$ by setting

$$
\begin{gathered}
\nabla_{S}(z \otimes 1)=0, \quad z \in S \\
\nabla_{S}\left(z \otimes x_{0} \wedge \ldots \wedge x_{p}\right)=\sum_{i=0}^{p}(-1)^{i-q} z \sigma\left(x_{i}\right) \otimes x_{0} \wedge \ldots \wedge \widehat{x}_{i} \wedge \ldots \wedge x_{p}
\end{gathered}
$$

(here and below the "hat" denotes omission of an element).

Some direct calculations imply that $S \otimes \wedge P$ becomes a graded differential algebra if one defines the multiplication in $S \otimes \wedge P$ by

$(z \otimes \Phi) \cdot(w \otimes \Psi)=(-1)^{p q} z w \otimes \Phi \Psi, \quad z \in S, w \in S^{q}, \Phi \in \bigwedge^{p} P, \Psi \in \wedge P$.

This graded differential algebra is called the Koszul complex.

Let $Q$ be an evenly graded finite-dimensional vector space with $Q^{k}=0$ for $k \leq 0$. Let $\bigvee Q$ be the appropriate symmetric $P$-algebra endowed with the induced grading

$$
\operatorname{deg}\left(y_{1} \vee \ldots \vee y_{q}\right)=\operatorname{deg}\left(y_{1}\right)+\ldots+\operatorname{deg}\left(y_{q}\right) .
$$

Definition. A $P$-algebra $(S, \sigma)$ with $S=\bigvee Q$ is called a symmetric P-algebra.

Definition. Let $R$ be any ring. A sequence $a_{1}, a_{2}, \ldots$ in $R$ is called regular if no $a_{i}$ is a zero divisor in the factor-ring $R /\left(a_{1}, \ldots, a_{i-1}\right)$. 
Theorem 2.1 [4]. Let $\left(\bigvee Q \otimes \wedge P, \nabla_{\sigma}\right)$ be a Koszul complex associated with a symmetric $P$-algebra. Let $y_{1}, \ldots, y_{n}$ be a basis of $P$ and let $s=\operatorname{dim} Q$. Suppose that $H^{*}\left(\bigvee Q \otimes \wedge P, \nabla_{\sigma}\right)$ is finite-dimensional. Then the minimal model $\left(\bigvee Q \otimes \wedge P, \nabla_{\sigma}\right)$ is formal if and only if the following conditions are satisfied:

(i) $\operatorname{dim} P=n \geq \operatorname{dim} Q=s$,

(ii) $\nabla_{\sigma}\left(y_{1}\right), \ldots, \nabla_{\sigma}\left(y_{s}\right)$ constitute a regular sequence in $\bigvee Q$ and

$$
\nabla_{\sigma}\left(y_{s+1}\right), \ldots, \nabla_{\sigma}\left(y_{n}\right) \in\left(\nabla_{\sigma}\left(y_{1}\right), \ldots, \nabla_{\sigma}\left(y_{s}\right)\right)
$$

after re-ordering if necessary.

Now, apply the above theorem to the case of homogeneous spaces of compact Lie groups. Recall the notion of the Cartan algebra of a homogeneous space. We consider compact Lie groups. To any compact homogeneous space $G / H$ one can assign a graded differential algebra $\left(C, \nabla_{\sigma}\right) \in \mathbb{R}-\mathrm{DGA}_{(\mathrm{c})}$ by the procedure described below. Let $T, T^{\prime}$ be maximal tori in $G$ and $H$ respectively $\left(T \supset T^{\prime}\right)$. Denote by $W(G)$ and $W(H)$ the Weyl groups associated with $T$ and $T^{\prime}$ and consider the corresponding $W(G)$ - and $W(H)$-actions on the Lie algebras $\tau$ and $\tau^{\prime}$ of $T$ and $T^{\prime}$. These actions are extended in a natural way to actions on the polynomial algebras $\mathbb{R}[\tau]$ nad $\mathbb{R}\left[\tau^{\prime}\right]$ :

$$
\sigma(f)(x)=f\left(\sigma^{-1}(x)\right)
$$

for any $\sigma \in W(G)$ (resp. $W(H)$ ), $f \in \mathbb{R}[\tau]$ (resp. $\mathbb{R}\left[\tau^{\prime}\right]$ ), $x \in \tau$ (resp. $\left.x \in \tau^{\prime}\right)$. Let $\mathbb{R}[\tau]^{W(G)}$ and $\mathbb{R}\left[\tau^{\prime}\right]^{W(H)}$ be the subalgebras of $W(G)$ - and $W(H)$-invariants. By the Chevalley theorem,

$$
\begin{aligned}
\mathbb{R}[\tau]^{W(G)} \simeq \mathbb{R}\left[f_{1}, \ldots, f_{n}\right], \quad n=\operatorname{rank} G, \\
\mathbb{R}\left[\tau^{\prime}\right]^{W(H)} \simeq \mathbb{R}\left[u_{1}, \ldots, u_{s}\right], \quad s=\operatorname{rank} H .
\end{aligned}
$$

Consider the usual representation of the cohomology algebra $H^{*}(G, \mathbb{R})$ as the exterior algebra over the primitive elements

$$
H^{*}(G, \mathbb{R}) \simeq \bigwedge\left(y_{1}, \ldots, y_{n}\right) .
$$

Define

$$
\begin{aligned}
& \left(C, \nabla_{\sigma}\right)=\left(\mathbb{R}\left[u_{1}, \ldots, u_{s}\right] \otimes \bigwedge\left(y_{1}, \ldots, y_{n}\right), \nabla_{\sigma}\right), \\
& \nabla_{\sigma}\left(u_{i}\right)=0, \quad i=1, \ldots, s, \\
& \nabla_{\sigma}\left(y_{j}\right)=\left.f_{j}\right|_{\tau^{\prime}}=\widetilde{f}_{j}\left(u_{1}, \ldots, u_{s}\right), \quad j=1, \ldots, n .
\end{aligned}
$$

Definition. The algebra $\left(C, \nabla_{\sigma}\right)$ defined by (1) is called the Cartan algebra of the homogeneous space $G / H$.

Obviously, $\left(C, \nabla_{\sigma}\right)$ is a particular example of the Koszul complex (however, in order to do so, one should associate with the corresponding sym- 
metric part a new grading by assigning to each $q \in Q=\left\langle u_{1}, \ldots, u_{s}\right\rangle$ the degree $2 \operatorname{deg}(q))$.

THEOREM 2.2 [5]. We have the following isomorphism:

$$
\mathcal{M}_{G / H} \simeq \mathcal{M}_{\left(C, \nabla_{\sigma}\right)} .
$$

TheOREM 2.3 [4]. Let $M=G / H$ be a homogeneous space of a compact Lie group $G$. Let $\left(C, \nabla_{\sigma}\right)$ be its Cartan algebra determined by $(1)$. Then $M$ is formal if and only if the sequence $\widetilde{f}_{1}, \ldots, \widetilde{f}_{n}$ satisfies the conditions (in an appropriate ordering)

(i) $\widetilde{f}_{1}, \ldots, \widetilde{f}_{s}$ constitute a regular sequence,

(ii) $\widetilde{f}_{s+1}, \ldots, \widetilde{f}_{n} \in\left(\widetilde{f}_{1}, \ldots, \widetilde{f}_{s}\right)$.

In [9] the notion of a pure graded differential algebra was introduced. By definition, a free graded differential algebra $(\bigwedge V, d)=\left(\bigwedge V^{\text {even }} \otimes \wedge V^{\text {odd }}, d\right)$ is called pure if $\left.d\right|_{V^{\text {even }}}=0, d\left(V^{\text {odd }}\right) \subset \wedge V^{\text {even }}$. Observe that this definition is stronger than the definition in [11] and Theorem 2.4 does not follow from [11].

Theorem 2.4. Let $\left(S, d_{S}\right)=\left(\bigvee Q \otimes \wedge P, d_{S}\right)$ and $\left(T, d_{T}\right)=\left(\bigvee Q^{\prime} \otimes \bigwedge P^{\prime}\right)$ be two Koszul complexes associated with symmetric $P$-algebras. Let $\tau$ be any "transgression" map $\tau: P^{\prime} \rightarrow Z(S)$ such that $d_{\tau}: S \otimes T \rightarrow S \otimes T$ defined by the formulae

$$
\left.d_{\tau}\right|_{S}=d_{S},\left.\quad d_{\tau}\right|_{Q^{\prime}}=0,\left.\quad d_{\tau}\right|_{P^{\prime}}=\left.d_{T}\right|_{P^{\prime}}+\left.\tau\right|_{P^{\prime}}
$$

is a derivation. Suppose that

$$
\operatorname{dim} Q^{\prime}=\operatorname{dim} P^{\prime} .
$$

Then if the twisted tensor product $\left(S \otimes_{\tau} T, d_{\tau}\right)$ has finite-dimensional cohomology algebra $H^{*}\left(S \otimes_{\tau} T, d_{\tau}\right)$, then it is pure.

Pr o of. Suppose that $\left(S \otimes_{\tau} T, d_{\tau}\right)$ is not pure. Choose a basis $\left(y_{1}^{\prime}, \ldots, y_{m}^{\prime}\right)$ in $P^{\prime}$ and $\left(y_{1}, \ldots, y_{n}\right)$ in $P$. From (2) there exists at least one basic element $y_{j}^{\prime}$ such that

$$
\tau\left(y_{j}^{\prime}\right)=\sum_{i_{1}, \ldots, i_{s}} h_{j}^{i_{1} \ldots i_{s}} y_{i_{1}} \wedge \ldots \wedge y_{i_{s}} \in Z_{+}\left(S \otimes_{\tau} T, d_{\tau}\right) .
$$

Since $\left.d_{\tau}\right|_{Q^{\prime}}=0$, the elements $f_{j}^{\prime}=d_{T}\left(y_{j}^{\prime}\right)$ and $\tau\left(y_{j}^{\prime}\right)$ are cohomologically equivalent:

$$
\left[f_{j}^{\prime}\right]=\left[\sum_{i_{1}, \ldots, i_{s}} h_{j}^{i_{1} \ldots i_{s}} y_{i_{1}} \wedge \ldots \wedge y_{i_{s}}\right]
$$

in $H^{*}\left(S \otimes_{\tau} T, d_{\tau}\right)$. From Theorem 2.1, $f_{1}^{\prime}, \ldots, f_{n}^{\prime}$ is a regular sequence in $\bigvee Q^{\prime}$, since $\left(T, d_{T}\right)$ is formal and $\operatorname{dim} Q^{\prime}=\operatorname{dim} P^{\prime}$. Therefore, $h f_{j}^{\prime} \notin$ $\left(f_{1}^{\prime}, \ldots, \widehat{f}_{j}^{\prime}, \ldots, f_{n}^{\prime}\right)$ for $h \in \bigvee Q^{\prime}$ if $h \notin\left(f_{1}^{\prime}, \ldots, \widehat{f}_{j}^{\prime}, \ldots, f_{n}^{\prime}\right)$. Moreover, the 
elements $h f_{j}^{\prime}$ cannot be coboundaries in $S \otimes_{\tau} T$, because (3) implies that the only possibility for $h f_{j}^{\prime}$ to be a coboundary is the following:

$$
h f_{j}^{\prime}=d_{\tau}\left(\sum_{j_{1}, \ldots, j_{k}} g_{j}^{j_{1} \ldots j_{k}} y_{j_{1}}^{\prime} \wedge \ldots \wedge y_{j_{k}}^{\prime}\right)
$$

( $S \otimes_{\tau} T$ is a free algebra and any term from $S$ will either remain in $S$ after differentiation, or will become zero). The latter formula shows that the only possibility which may occur is $k=1$, which yields

$$
h f_{j}=d_{\tau}\left(\sum_{q} g_{j}^{q} y_{q}^{\prime}\right) \text {. }
$$

Consider all $y_{j}^{\prime}$ for which $\tau\left(y_{j}^{\prime}\right) \in Z_{+}\left(S \otimes_{\tau} T, d_{\tau}\right)$. Without loss of generality one can assume that all expressions (3) either differ by variables $y_{i_{1}} \wedge \ldots \wedge y_{i_{s}}$, or $h_{j}^{i_{1} \ldots i_{s}}$ are linearly independent; otherwise one could obtain, for example,

$$
\begin{aligned}
& d_{\tau}\left(y_{p}^{\prime}\right)=f_{p}^{\prime}+\sum h_{p}^{i_{1} \ldots i_{s}} y_{i_{1}} \wedge \ldots \wedge y_{i_{s}}, \\
& d_{\tau}\left(y_{q}^{\prime}\right)=f_{q}^{\prime}+\sum h_{q}^{i_{1} \ldots i_{s}} y_{i_{1}} \wedge \ldots \wedge y_{i_{s}},
\end{aligned}
$$

with $h_{q}^{i_{1} \ldots i_{s}}=\mu h_{p}^{i_{1} \ldots i_{s}}$ and $d_{\tau}\left(y_{q}^{\prime}-\mu y_{p}^{\prime}\right)=f_{q}^{\prime}-\mu f_{p}^{\prime}$, and making an appropriate change of variables one obtains $\tau\left(y_{q}^{\prime}\right) \in Z_{0}\left(S \otimes_{\tau} T\right)$, lowering the number of variables whose image is contained in $Z_{+}\left(S \otimes_{\tau} T\right)$. Thus, (4) can be rewritten as

$$
h f_{j}^{\prime}=d_{\tau}\left(\sum_{q} g_{j}^{q} y_{q}^{\prime}\right)=\sum_{q} f_{q}^{\prime} g_{j}^{q}+\sum_{q}\left(\sum h_{q}^{i_{1} \ldots i_{s}} y_{i_{1}} \wedge \ldots \wedge y_{i_{s}}\right) g_{j}^{q} .
$$

Using the conditions of freeness, one obtains

$$
\sum_{l} h_{l}^{i_{1} \ldots i_{s}} \otimes g_{j}^{l}=0, \quad h_{l}^{i_{1} \ldots i_{s}} \in \bigvee Q, g_{j}^{l} \in \bigvee Q^{\prime}
$$

where by assumption $h_{l}^{i_{1} \ldots i_{s}}$ are linearly independent. Thus, $g_{j}^{q}=0$. Therefore, finally

$$
h f_{j}^{\prime} \in\left(f_{1}^{\prime}, \ldots, \widehat{f}^{\prime}{ }_{j}, \ldots, f_{n}^{\prime}\right)
$$

(because $g_{j}^{j}$ certainly belongs to the set $\left\{g_{j}^{l}\right\}$ ). Since $f_{1}^{\prime}, \ldots, f_{n}^{\prime}$ is a regular sequence, $h \in\left(f_{1}^{\prime}, \ldots, \widehat{f}^{\prime}{ }_{j}, \ldots, f_{n}^{\prime}\right)$. Since $\operatorname{dim} Q^{\prime}=n$, one can find an infinite sequence of polynomials $h_{\alpha}, \alpha=1,2, \ldots$, with $h_{\alpha} \notin\left(f_{1}^{\prime}, \ldots, \widehat{f}_{j}^{\prime}, \ldots, f_{n}^{\prime}\right)$. Therefore, the latter implies the existence of an infinite sequence of linearly independent cohomology classes $\left[h_{\alpha} f_{j}\right], \alpha=1,2, \ldots$, in $H^{*}\left(S \otimes_{\tau} T, d_{\tau}\right)$, which is a contradiction. The theorem is proved.

3. Fat bundles. Recall the construction of fat bundles invented by A. Weinstein [14]. The basic notions of symplectic geometry can be found 
in [12]. Let $P(M, G)$ be a smooth principal bundle with connection form $\theta$ and curvature form $\Omega$ (see [7]). Let $\mathcal{H}$ and $\mathcal{V}$ denote respectively the horizontal and vertical distributions in TP. Since $\Omega$ is a tensorial form on $P$ which is $\operatorname{Ad} G$-invariant and $g$-valued, we obtain horizontal, scalar-valued forms on $P$ by taking compositions $\mu \circ \Omega, \mu \in g^{*}$. If $S \subset g^{*}$ is any subset, we call the given connection $S$-fat if, for every $\mu \in S$, the form $\mu \circ \Omega$ is nondegenerate on $\mathcal{H}$ in $T P$. In particular, let $\mathcal{O} \subset g^{*}$ be a co-adjoint orbit. We say that the given connection is $\mathcal{O}-f a t$ if $\mu \circ \Omega$ is nondegenerate for any $\mu \in \mathcal{O}$.

TheOREM 3.1 [14]. Let $E=P \times{ }_{G} \mathcal{O}$ be the total space of the associated bundle of any principal bundle $P(M, G)$, equipped with an $\mathcal{O}$-fat connection. Then $E$ is a symplectic manifold.

Proof. Let $\omega$ denote the symplectic form on $\mathcal{O}$ and $\pi_{1}$ denote the projection $P \times \mathcal{O} \rightarrow \mathcal{O}$. Consider the 1 -form on $P \times \mathcal{O}$ defined as follows:

$$
\langle\theta\rangle_{(u, \mu)}\left(X_{P} \oplus X_{\mathcal{O}}\right)=\mu\left(\theta_{u}\left(X_{P}\right)\right)
$$

where $\mu \in \mathcal{O}, X_{P}$ is a vector field on $P$ and $X_{\mathcal{O}}$ is a vector field on $\mathcal{O}$. The following equality can be verified directly:

$$
d\langle\theta\rangle(\widetilde{X}, \widetilde{Y})=\langle d \theta\rangle(\widetilde{X}, \widetilde{Y})-\widetilde{X}\langle\theta\rangle(\widetilde{Y})+\widetilde{Y}\langle\theta\rangle(\widetilde{X})
$$

for any vector fields $\widetilde{X}, \widetilde{Y}$ on $P \times \mathcal{O}$.

Let $\bar{X}=\bar{X}_{P}+\bar{X}_{F}$ denote the fundamental vector field on $P \times \mathcal{O}$. Then for any vector field $Y$ on $P \times \mathcal{O}$,

$$
d\langle\theta\rangle(\bar{X}, Y)+\pi_{1}^{*} \omega(\bar{X}, Y)=\omega\left(\bar{X}_{F},\left(\pi_{1}\right)_{*}(Y)\right)-\omega\left(\bar{X}_{F},\left(\pi_{1}\right)_{*}(Y)\right)=0 .
$$

Now, it is easy to verify that both $\langle\theta\rangle$ and $\omega$ are $G$-invariant:

$$
\begin{aligned}
\langle\theta\rangle_{\left(R_{a}(u), \operatorname{Ad}_{a-1}^{*}(\mu)\right)} & =\operatorname{Ad}_{a^{-1}}^{*}(\mu) \circ \theta_{R_{a}(u)}\left(R_{a}\right)_{*} \\
& =\mu \circ \operatorname{Ad}_{a}\left(\theta_{R_{a}(u)}\left(R_{a}\right)_{*}\right)=\mu \circ\left(R_{a^{-1}}\right)^{*} \theta_{R_{a}(u)}\left(R_{a}\right)_{*} \\
& =\mu\left(\theta_{u}\right)=\langle\theta\rangle_{(u, \mu)} .
\end{aligned}
$$

Because $\omega$ is $G$-invariant, the same is valid for $d\langle\theta\rangle+\pi_{1}^{*} \omega$. Hence there exists a unique 2-form $\omega_{E}$ defined on $E$ by the equality

$$
d\langle\theta\rangle+\pi_{1}^{*} \omega=\pi^{*} \omega_{E}
$$

where $\pi: P \times \mathcal{O} \rightarrow E$ is the natural projection. Because $d\langle\theta\rangle+\pi_{1}^{*} \omega$ is obviously closed, one can deduce that $d \omega_{E}=0$. We shall prove that the following equality holds:

$$
\left.\left(d\langle\theta\rangle+\pi_{1}^{*} \omega\right)\right|_{\mathcal{H} \oplus T \mathcal{O}}=\left.\left(\langle\Omega\rangle+\pi_{1}^{*} \omega\right)\right|_{\mathcal{H} \oplus T \mathcal{O}}
$$

(here $\langle\Omega\rangle$ is defined by the same rule (6)). The 2-form $\left.\left(\langle\Omega\rangle+\pi_{1}^{*} \omega\right)\right|_{\mathcal{H} \oplus T \mathcal{O}}$ is nondegenerate by the definition of fatness. After the identification $\mathcal{H}_{u} \oplus$ $T_{\mu} \mathcal{O} \simeq T_{\pi(u, \mu)} E$, we conclude that $\omega_{E}$ is nondegenerate. 
Remark. It is not evident why the 2 -form (7) is closed. Since there is no proof of this fact in [14], we give it here for the convenience of the reader.

There are some natural obstructions to fatness [14]. For example, $M$ must be even-dimensional and orientable, and some real characteristic numbers of $P(M, G)$ must be nonzero. In the next section we investigate the same question for homogeneous spaces $M=H / K$ and show that they must be formal.

4. Minimal models of fat bundles. We begin with a theorem which is contained in [5] and [11].

TheOREm $4.1[5]$. Let $P(M, G)$ be a principal bundle, let $h_{P}: V g^{*} \rightarrow$ $H^{*}(M)$ be its Weil homomorphism, and let $\tau: \bigwedge g^{*} \rightarrow \bigvee g^{*}$ be the transgression mapping determined by the Lie group $G$. Let $U$ be a closed subgroup in $G$ and let $\left(C_{G / U}, \delta_{G / U}\right)$ be the Cartan algebra of $G / U$. Consider the associated bundle $E(G / U, M, P)$. Then the following c-equivalence of graded differential algebras is valid:

$$
\begin{gathered}
\left(\mathcal{E}_{E}, d_{\mathcal{E}_{E}}\right) \sim^{c}\left(\mathcal{E}_{M} \otimes C_{G / U}, \delta\right), \\
\left.\delta\right|_{\mathcal{E}_{M}}=d_{\mathcal{E}_{M}}, \quad \delta\left(u_{i}\right)=0, \quad i=1, \ldots, s, \\
\delta\left(y_{j}\right)=\delta_{G / U}\left(y_{j}\right)+\gamma_{M}\left(y_{j}\right), \quad j=1, \ldots, n,
\end{gathered}
$$

where $\gamma_{M}\left(y_{j}\right)$ denote any closed elements representing the cohomology classes $h_{P}\left(\tau\left(h_{j}\right)\right)$ in $H^{*}(M)$.

The proof can be found in [5] (volume 3) (see also [11]).

Corollary. Let $P(M, G)$ and $E(M, P, G, G / U)$ be as in Theorem 4.1. Suppose that $M=H / K$ is also a homogeneous space of a compact Lie group $H$. Then the minimal model $\mathcal{M}_{E}$ is determined by the isomorphism

$$
\mathcal{M}_{E} \simeq \mathcal{M}_{\left(\mathcal{A}, d_{\mathcal{A}}\right)}
$$

where $\left(\mathcal{A}, d_{\mathcal{A}}\right)$ is of the form

$$
\begin{aligned}
\left(\mathcal{A}, d_{\mathcal{A}}\right) & =\left(C_{H / K} \otimes C_{G / U}, d_{\mathcal{A}}\right), \\
\left.d_{\mathcal{A}}\right|_{C_{H / K}} & =\delta_{H / K}, \quad d_{\mathcal{A}}\left(u_{i}\right)=0, \quad i=1, \ldots, s, \\
d_{\mathcal{A}}\left(y_{j}\right) & =\delta_{G / U}\left(y_{j}\right)+\widetilde{\gamma}_{M}\left(y_{j}\right), \quad j=1, \ldots, n,
\end{aligned}
$$

and $\widetilde{\gamma}_{M}\left(y_{j}\right)$ denote closed elements in $C_{H / K}$ representing the cohomology classes in $H^{*}\left(C_{H / K}\right)=H^{*}(M)$ such that $\alpha^{*}\left[\tilde{\gamma}_{M}\left(y_{j}\right)\right]=\left[\gamma_{M}\left(y_{j}\right)\right]$, where $\alpha^{*}$ is induced by an appropriate quasiisomorphism $\alpha: C_{H / K} \rightarrow \mathcal{E}_{M}$.

Proof. From Theorem $4.1,\left(\mathcal{E}_{E}, d_{\mathcal{E}_{E}}\right)$ is c-equivalent to $\left(\mathcal{E}_{M} \otimes C_{G / U}, \delta\right)$. It is easy to see that this $c$-equivalence can be continued:

$$
\left(\mathcal{E}_{M} \otimes C_{G / U}, \delta\right) \sim\left(\mathcal{E}_{M} \otimes \mathcal{M}_{G / U}, \bar{\delta}\right)
$$


where $\mathcal{M}_{G / U}$ is the minimal model for $C_{G / U}$ which can be represented in the form

$$
\mathcal{M}_{G / U}=k\left[v_{1}, \ldots, v_{t}\right] \otimes \bigwedge\left(\bar{y}_{1}, \ldots, \bar{y}_{l}\right) .
$$

Here $\bar{y}_{1}, \ldots, \bar{y}_{l}$ correspond canonically to $y_{i_{1}}, \ldots, y_{i_{l}}$ and $\bar{\delta}\left(v_{i}\right)=0, i=$ $1, \ldots, t, \bar{\delta}\left(\bar{y}_{j}\right)=\bar{f}_{j}\left(v_{1}, \ldots, v_{t}\right)+\bar{\gamma}_{M}\left(\bar{y}_{j}\right)$, where $\bar{\gamma}_{M}\left(\bar{y}_{j}\right)=\pi \gamma_{M}\left(y_{j}\right)$ for the canonical projection $\pi: C_{G / U} \rightarrow \mathcal{M}_{G / U}$, given by Sullivan's algorithm [8]. The definition of $\bar{\delta}$ implies that the natural projection

$$
\left(\mathcal{E}_{M} \otimes C_{G / U}, \delta\right) \rightarrow\left(\mathcal{E}_{M} \otimes \mathcal{M}_{G / U}, \bar{\delta}\right)
$$

is a quasiisomorphism (this is verified by direct calculation). Thus, finally, one obtains the following diagram of $K S$-extensions:

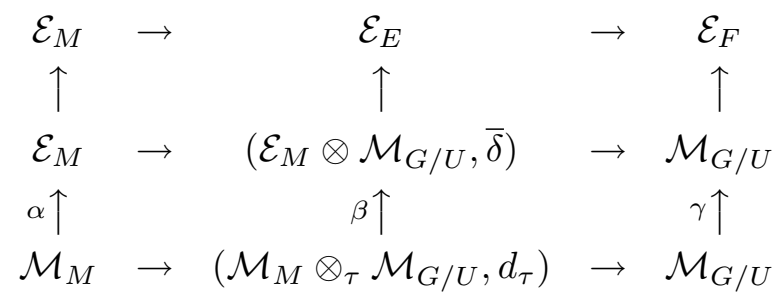

where all vertical arrows are quasiisomorphisms, $\gamma$ is an isomorphism, the horizontal arrows are inclusions or projections defined by augmentations. The existence of the first two lines of the diagram is proven by Grivel, Thomas [11] and Halperin [6]. If one defines

$$
\left.d_{\tau}\right|_{\mathcal{M}_{M}}=d_{\mathcal{M}_{M}}, \quad d_{\tau}\left(\bar{y}_{j}\right)=\gamma^{-1}\left(f_{j}\left(v_{1}, \ldots, v_{t}\right)+\widetilde{\gamma}_{M}\left(\bar{y}_{j}\right), d_{\tau}\left(\bar{v}_{i}\right)=0\right.
$$

where $\alpha^{*}\left[\widetilde{\gamma}_{M}\left(\bar{y}_{j}\right)\right]=\left[\widetilde{\gamma}_{M}\left(\bar{y}_{j}\right)\right]$, then $\beta$ becomes a morphism of DGA, inducing isomorphism in cohomology by the 5-lemma. Therefore $d_{\tau}$ really can be chosen in the form given above and $\left(\mathcal{M}_{M} \otimes \mathcal{M}_{G / U}, d_{\tau}\right)$ is a model for $E$. The same argument shows that $\left(\mathcal{M}_{M} \otimes \mathcal{M}_{G / U}, d_{\tau}\right)$ is a model for $\left(\mathcal{A}, d_{\mathcal{A}}\right)$, and (8) follows.

Now we can give the proof of Theorem 1.1.

Proof of Theorem 1.1. Since the orbit $\mathcal{O}$ is a homogeneous $G$ manifold, say, $G / U, E=P \times_{G} G / U$ satisfies the conditions of Theorem 4.1. Therefore, from the previous corollary, one should consider the formality problem for the minimal model of the algebra $\left(\mathcal{A}, d_{\mathcal{A}}\right)$ given by $(8)$. The latter algebra is a twisted tensor product of two symmetric $P$-algebras. Since $\mathcal{O}$ is a homogeneous symplectic manifold of a compact Lie group $G, \mathcal{O}$ is a homogeneous space $G / U$ of maximal rank (moreover, $U$ is the centralizer of a torus, which follows from Borel's theorem [15]). Therefore, $\mathcal{O}$ is formal and thus the second term in the twisted tensor product representing $\left(\mathcal{A}, d_{\mathcal{A}}\right)$ satisfies the conditions of Theorem 2.4. Since $E$ is symplectic by Theorem 3.1, $\mathcal{M}_{E}$ is a symplectic minimal algebra in the sense of [9] (there exists an el- 
ement $\omega \in \mathcal{M}_{E}$ of degree 2 such that on the cohomology level $\omega^{k} \neq 0$ for any $k=1, \ldots, n, \operatorname{dim} E=2 n)$. The following theorem was proved in [9].

TheOREM 4.2. Any symplectic pure minimal algebra satisfying the Poincaré duality is formal and has only even-dimensional cohomology.

Applying this theorem together with Theorem 2.4 to $\left(\mathcal{A}, d_{\mathcal{A}}\right)$ (or, to be more precise, to $\left.\mathcal{M}_{\left(\mathcal{A}, d_{\mathcal{A}}\right)}\right)$, one finds that $\mathcal{M}_{\left(\mathcal{A}, d_{\mathcal{A}}\right)} \simeq \mathcal{M}_{E}$ is formal.

It remains to show that $M$ is formal and of maximal rank. Since $\left(\mathcal{A}, d_{\mathcal{A}}\right)$ is pure by Theorem 2.4, it is again a Koszul complex: for example, of the form $\left(\bigvee Q \otimes \bigvee Q^{\prime} \otimes \wedge P \otimes \bigwedge P^{\prime}, \nabla\right)$. Thus, the following two possibilities may occur (since $\operatorname{dim} Q^{\prime}=\operatorname{dim} P^{\prime}$ and $\left(\bigvee Q^{\prime} \otimes \bigwedge P^{\prime}, \nabla_{\sigma_{1}}\right)$ is formal).

(1) $\operatorname{dim} P>\operatorname{dim} Q$ (equivalently, $\operatorname{rank} H>\operatorname{rank} K$ ),

(2) $\operatorname{dim} P=\operatorname{dim} Q$ (equivalently, $\operatorname{rank} H=\operatorname{rank} K, M$ is formal).

Thus, one only has to consider the first possibility. Then, considering $\left(\bigvee Q \otimes \bigvee Q^{\prime} \otimes \bigwedge P^{\prime} \otimes \bigwedge P, \nabla\right)$ as a new Koszul complex, one can apply Theorem 2.1 to it and deduce that either its minimal model is not formal (which is a contradiction), or the sequence of $\nabla y_{1}, \ldots, \nabla y_{1}^{\prime}, \ldots$ satisfies the condition (i) of Theorem 2.1 with $s=\operatorname{dim} Q+\operatorname{dim} Q^{\prime}$. The latter equality together with the proof of Theorem 2.1 show that there are odd-degree elements in $H^{*}(E)=H^{*}\left(\bigvee Q \otimes \bigvee Q^{\prime} \otimes \bigwedge P^{\prime} \otimes \bigwedge P, \nabla\right)$ which contradicts Theorem 4.2 .

Remark. The proof of Theorem 2.1 is contained in [4].

5. Existence of $\mathcal{O}$-fat connections. There are examples of various fatness conditions in [2] and [13]. Nevertheless it is still interesting and important to give explicit examples of $\mathcal{O}$-fatness to be able to construct new symplectic structures. Besides that it is rather obvious that for any $G$-principal bundle, any homomorphism

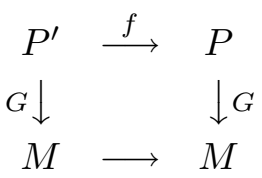

can only decrease the rank of the appropriate 2-form, that is,

$$
\left.\operatorname{rank} \mu \circ f^{*} \Omega\right|_{\mathcal{H}^{\prime}} \leq\left.\operatorname{rank} \mu \circ \Omega\right|_{\mathcal{H}}
$$

Therefore, one can obtain an $\mathcal{O}$-fat connection only in the case of its existence in the universal G-principal bundle. It is known that for sufficiently large $m$, Stiefel manifolds over Grassmannians give universal $S O(n)$-bundles (see $[10])$. 
THEOREM 5.1. Let

$$
S(2 m+2 n, 2 m, 2 n) \stackrel{S O(2 n)}{\longrightarrow} \operatorname{Gr}(2 m+2 n, 2 m, 2 n)
$$

denote the universal $S O(2 n)$-bundle of a Stiefel manifold $S O(2 m+2 n)$ / $S O(2 m)$ over a Grassmann manifold $S O(2 m+2 n) / S O(2 m) \times S O(2 n)$, $n>1$. There exists a co-adjoint orbit $\mathcal{O}(\xi) \subset s o(2 n)^{*}$ such that the canonical connection of the Riemannian symmetric space $\operatorname{Gr}(2 m+2 n, 2 m, 2 n)$ is $\mathcal{O}(\xi)-f a t$.

Proof. The bundle (9) can be considered as a particular case of a $G$-structure over a Riemannian homogeneous symmetric space $H / K$. It is known [7] that there is a one-to-one correspondence between invariant connections in $P(H / K, G)$ and $\operatorname{Ad}(H)$-invariant linear maps

$$
\Lambda: \mathcal{X} \rightarrow g,
$$

where $\mathcal{X}$ is a reductive complement to $k$ in $h$. In our particular case $g=$ $s o(2 n), h=s o(2 m+2 n), k=s o(2 m) \oplus s o(2 n), \mathcal{X}$ consists of matrices

$$
X=\left(\begin{array}{cc}
0 & A \\
-A^{t} & 0
\end{array}\right)
$$

( $A$ is any $2 m \times 2 n$ matrix). It is known [7] that $\Lambda \equiv 0$ for the canonical connection. Recall that the correspondence is given by the formula

$$
\theta_{u}(\widetilde{X})=\Lambda(X)
$$

where $\widetilde{X}$ is the horizontal lift of $X$. Thus, using the usual formula [7] for the curvature form

$$
2 \Omega(\widetilde{X}, \widetilde{Y})=\theta([\widetilde{X}, \widetilde{Y}])+[\theta(\widetilde{X}), \theta(\widetilde{Y})]
$$

and (11) one easily calculates

$$
\Omega(\tilde{X}, \widetilde{Y})=\frac{1}{2}[X, Y]_{s o(2 n)}
$$

where $X$ and $Y$ are of the form (10). Finally,

$$
\Omega(\widetilde{X}, \tilde{Y})=B^{t} A-A^{t} B
$$

where $A$ and $B$ are $2 m \times 2 n$ matrices representing $X$ and $Y$. Therefore, if $\xi \in s o(2 n)^{*}$, then $\left.\xi \circ \Omega\right|_{\mathcal{H}}$ is identified with the skew symmetric bilinear map

$$
\widetilde{\xi}: V \times V \rightarrow \mathbb{R}, \quad \widetilde{\xi}(A, B)=\xi\left(B^{t} A-A^{t} B\right),
$$

where $V$ is a $2 m \cdot 2 n$-dimensional space of $2 m \times 2 n$ matrices. Suppose that the functional $\eta \in \mathcal{O}(\xi)$. Then one obtains

$$
\widetilde{\eta}(A, B)=\xi\left(S^{t}\left(B^{t} A-A^{t} B\right) S\right), \quad S \in S O(2 n)
$$

(this comes from the definition of the co-adjoint action). Now take the standard basis of $V$ consisting of the matrices $e_{i j}, 1 \leq i \leq 2 m, 1 \leq j \leq 2 n$. Let $s_{l k}$ be the standard basis of $s o(2 n), 1 \leq l \leq 2 n, 1 \leq k \leq 2 n, l<k$, 
consisting of $n(n-1) / 2$ skew-symmetric matrices with 1 at position $(l, k)$ and other elements zero. Define $\xi \in s o(2 n)^{*}$ as the functional

$$
\xi\left(s_{l k}\right)=1, \quad 1 \leq l \leq 2 n, 1 \leq k \leq 2 n, l<k .
$$

Then calculating $\widetilde{\xi}$ by the formula (11) one obtains the $2 m \cdot 2 n \times 2 m \cdot 2 n$ matrix of $\widetilde{\xi}$

$$
U_{\tilde{\xi}}=\left(\begin{array}{cccc}
U & 0 & \ldots & 0 \\
0 & U & \ldots & 0 \\
\ldots & \ldots \ldots & \ldots \\
0 & 0 & \ldots & 0 \\
0 & 0 & \ldots & U
\end{array}\right)
$$

( $2 m$ blocks $U$ on the diagonal), where $U$ is a skew-symmetric matrix with

$$
u_{i j}=-1, \quad i<j, 1 \leq i \leq 2 n, 1 \leq j \leq 2 n .
$$

The matrix of the functional $\widetilde{\eta}$ with $\eta \in \mathcal{O}(\xi)$ given by (12) can be calculated as

$$
U_{\widetilde{\eta}}=\left(\begin{array}{cccc}
S U S^{t} & 0 & \ldots & 0 \\
0 & S U S^{t} & \ldots & 0 \\
\ldots \ldots \ldots \ldots \ldots \ldots \ldots \ldots \ldots \\
0 & 0 & \ldots & 0 \\
0 & 0 & \ldots & S U S^{t}
\end{array}\right)
$$

where $S \in S O(2 n)$ and $U$ is defined by (12). It remains to notice that $U$ is nondegenerate. Thus, the matrix of $\widetilde{\eta}$ is nondegenerate, which proves that the canonical connection is $\mathcal{O}$-fat.

Remark. If one considers $S O(n)$-bundles with $n$ odd, it is clear that the above argument does not work, since $U$ becomes degenerate.

Acknowledgements. The second author is grateful to GETODIM at Université Montpellier II for their hospitality and to the Polish KBN for financial support (research project no. 2P301 009 05).

\section{References}

[1] C. Allday and V. Puppe, Cohomology Theory of Transformation Groups, Cambridge Univ. Press, 1993.

[2] L. Bérard-Bergery, Sur certaines fibrations d'espaces homogènes riemanniennes, Compositio Math. 30 (1975), 43-61.

[3] P. Deligne, P. Griffiths, J. Morgan and D. Sullivan, Real homotopy theory of Kähler manifolds, Invent. Math. 29 (1975), 245-274.

[4] A. Dumańska-Małyszko, Z. Stępień and A. Tralle, Generalized symmetric spaces and minimal models, Ann. Polon. Math. 64 (1996), 17-35.

[5] V. Greub, S. Halperin and R. Vanstone, Connections, Curvature and Cohomology, Academic Press, New York, 1976. 
[6] S. Halperin, Lectures on Minimal Models, Hermann, Paris, 1982.

[7] S. Kobayashi and K. Nomizu, Foundations of Differential Geometry, Vol. 2, Interscience Publ., New York, 1969.

[8] D. Lehmann, Théorie homotopique des formes différentielles (d'après D. Sullivan), Astérisque 45 (1977).

[9] G. Lupton and J. Oprea, Symplectic manifolds and formality, J. Pure Appl. Algebra 91 (1994), 193-207.

[10] R. Narasimhan and S. Ramanan, Existence of universal connections, Amer. J. Math. 83 (1961), 536-572.

[11] J.-C. Thomas, Rational homotopy of Serre fibrations, Ann. Inst. Fourier (Grenoble) 31 (3) (1981), 71-90.

[12] I. Vaisman, Symplectic Geometry and Secondary Characteristic Classes, Birkhäuser, Basel, 1988.

[13] M. Vigué-Poirrier and D. Sullivan, Cohomology theory of the closed geodesic problem, J. Differential Geom. 11 (1976), 633-644.

[14] A. Weinstein, Fat bundles and symplectic manifolds, Adv. in Math. 37 (1980), 239-250.

[15] P. B. Zwart and W. M. Boothby, On compact, homogeneous symplectic manifolds, Ann. Inst. Fourier (Grenoble) 30 (1) (1980), 129-157.

Institute of Mathematics

University of Szczecin

Wielkopolska 15

70-451 Szczecin, Poland 